\title{
La formación de Trabajo Social en Túnez: aprendizajes y retos desde una experiencia de cooperación interuniversitaria
}

\author{
Social Work education in Tunisia: lessons and challenges \\ from an experience of university cooperation \\ Belén Morata-García de la Puerta \\ Universidad de Granada \\ bmorata@ugr.es \\ Mourad ABOUSSI \\ Universidad de Granada \\ maboussi@ugr.es \\ Eva $\mathrm{M}^{\mathrm{a}}$ JUAN TOSET \\ Universidad de Granada \\ ejuan@ugr.es
}

Recibido: 09/04/2014

Revisado: 29/05/2014

Aceptado: 03/11/2014

Disponible on line: 10/12/2014

\section{Resumen}

Este artículo tiene por objetivo describir un ejemplo de cooperación interuniversitaria, como modelo de buenas prácticas y de transferencia del conocimiento en el ámbito del Trabajo Social Internacional. El marco de referencia es la convocatoria pública que la Oficina Técnica de Cooperación de la AECID en Túnez realizó en el año 2012, del Proyecto de Apoyo al Instituto Nacional de Trabajo y Estudios Sociales-Túnez, para una asistencia técnica en la definición del plan de formación y estudios. En este trabajo se pretende resaltar la oportunidad de la cooperación interuniversitaria como una eficaz herramienta de colaboración horizontal entre instituciones de enseñanza superior. La adopción de metodologías participativas que priman la dimensión del inter-aprendizaje frente a la asistencia técnica de carácter vertical y el reconocimiento del liderazgo de los actores locales impregnan todas las fases del proyecto, no sólo el diagnóstico inicial. El objetivo resalta el apoyo para mejorar la definición del plan de formación y estudios del INTES y su adaptación tanto a los intereses de la comunidad académica como a los retos actuales de Túnez para reconstruir sus instituciones y políticas.

Palabras clave: Trabajo Social, cooperación interuniversitaria, Túnez, buenas prácticas, inter-aprendizaje.

\begin{abstract}
This article aims to describe an example of inter-university cooperation, as a model of good practices and knowledge transfer in the field of International Social Work. The framework is the public announcement conducted in 2012 by Technical Cooperation Office of the AECID in Tunisia. This was a Project to Support the National Institute of Labor and Social Studies-Tunisia, for technical assistance in the definition of the training plan and studies. This paper aims to highlight the opportunity of inter-university cooperation as an effective tool for horizontal collaboration among institutions of higher education. This project adopts in all his phases the participatory methodologies in order to give priority to share learning and to recognize the leadership of local actors, versus the vertical nature of technical assistance. The objective highlights the support to improve the definition of training plan and studies of the INTES, adapting both to the interests of the academic community and the current challenges of Tunisia to rebuild its institutions and policies.
\end{abstract}

Keywords: Social Work, inter-university cooperation, Tunisia, good practices, inter-learning.

Referencia normalizada: Morata-García de la Puerta, B., Abouss, M. y Juan Toset E. M. ${ }^{\text {a }}$ (2014): «La formación de Trabajo Social en Túnez: aprendizajes y retos desde una experiencia de cooperación interuniversitaria». Cuadernos de Trabajo Social, 27(2): 343-351.

Sumario: Introducción. 1. La formación en Trabajo Social: evolución y retos actuales. 2. Descubriendo de cerca la realidad del Trabajo Social en Túnez. 3. Fase de intercambio sobre Trabajo Social en Granada. 4. Conclusiones. 5. Referencias bibliográficas. 


\section{Introducción ${ }^{1}$}

Este artículo tiene por objetivo describir un ejemplo de cooperación interuniversitaria, como modelo de buenas prácticas y de transferencia del conocimiento en el ámbito del Trabajo Social internacional.

En el año 2012 la Oficina Técnica de Cooperación de la AECID en Túnez convocó, en el marco de la cooperación hispano-tunecina y en concurso público, un proyecto de cooperación para contribuir al desarrollo de las capacidades del Instituto Nacional de Trabajo y Estudios Sociales (INTES). La demanda se concretaba en el diseño de un plan de apoyo a la mejora de la formación dentro de dicho Instituto, tanto para el profesorado como para los/as trabajadores/as sociales en las unidades locales de promoción social. De igual manera, se resaltaba la idea de mejorar el sistema de prácticas profesionales para el alumnado del centro, así como aproximarse a nuevos (y viejos) problemas sociales.

El recurso a la cooperación fue, y sigue siendo, inevitable para los investigadores de Túnez, al excluir a la investigación científica del campo universitario de este país y arrastrar un concepto más restrictivo de «ciencia útil», en el que predominaba una visión puramente cuantitativa e institucional (Siino, 2004). En este sentido, la cooperación bilateral entre instituciones de educación superior se ha dirigido tradicionalmente a compartir experiencias y recursos que se puedan trasladar a los procesos de desarrollo en que cada Universidad se encuentre comprometida. En relación con ello, la O.C.D.E. atribuye a las Universidades un papel importante en la transferencia del conocimiento, y un protagonismo como agentes de desarrollo local y regional. Formar recursos humanos y dotarlos de las capacidades específicas para este campo de trabajo es una de las aportaciones a la cooperación, así como el estudio y la investigación sobre los que abordar los diagnósticos, sustentar las decisiones y fundamentar los programas de actuación de los actores.

Este encargo fue adjudicado al grupo de investigación SEPISE, que entendió el mismo co- mo un ejemplo de transferencia del conocimiento, al no ser privativo de las ciencias duras, y referirse a una forma de hacer las cosas que implicaban a la vez un objetivo, un modo y un saber (Castro Martínez, Fernández de Lucio, Pérez Marín y Criado Boado, 2008). Con anterioridad, el grupo ya había afrontado otros retos semejantes, de apoyo técnico a la formación universitaria de trabajadores/as sociales, en países emergentes y, en consecuencia, con experiencia en la coordinación y acompañamiento de proyectos internacionales en el ámbito del Trabajo Social. La investigación en torno a la formación, la innovación y las metodologías docentes; la inclusión de la diversidad y la construcción de estrategias didácticas para su tratamiento integrador, fueron algunos de los temas sobre los que el grupo tenía experiencia. Más que «un producto», la transferencia en tales casos se entendió como asesoramiento, consultoría, apoyo e investigación, siguiendo una metodología participativa y dejando clara la apuesta por la dimensión comparativa y dialógica en las cláusulas del convenio-contrato. En efecto, se acordaron procedimientos de participación inter pares y - con especial consideración- de comunicación multicultural basada en el reconocimiento mutuo y el respeto. Todas las fases del proyecto se diseñaron como propuestas del equipo del SEPISE sujetas a la aceptación de los profesores del Instituto Nacional de Trabajo y Estudios Sociales y, en su caso, adaptación al contexto que conocen y a las necesidades detectadas. Este enfoque deja atrás la asistencia técnica de carácter vertical y emana de un reconocimiento del liderazgo de los actores locales como parte fundamental de un modelo de cooperación horizontal que implica a los beneficiaros en todas las fases del proyecto, no sólo en el diagnóstico inicial.

La composición del grupo era también un valor, pues tenía una doble procedencia, académica y profesional, y se aunaba la experiencia en la enseñanza del Trabajo Social y la práctica. Se aportaba un modelo innovador de organización y gestión de la investigación que consistía

${ }^{1}$ El trabajo es resultado de un proyecto de cooperación interuniversitaria. El equipo estaba formado por: Enrique Raya Lozano como Investigador Principal, Belén Morata-García de la Puerta, Mourad Aboussi, Eva $M^{a}$ Juan-Toset, María Auxiliadora Trujillo Vega, María Luz Morante del Peral (Grupo de Investigación SEPISE-Universidad de Granada), Francesco Vacchiano (Universidad de Lisboa) y Marie Lacroix (Université de Montréal). 
en la articulación entre investigación y formación, tanto del personal investigador como de los profesionales en ejercicio, desde enfoques próximos a la reflexión en la acción. El objetivo fue desde el principio crear un espacio de trabajo compartido con el personal docente e investigador del Instituto Nacional de Trabajo y Estudios Sociales, con el resto de la comunidad académica (estudiantes, profesionales con vinculación a la institución), así como con los otros actores de lo social.

\section{La formación en Trabajo Social: evolu- ción y retos actuales}

El contexto social y político del Túnez contemporáneo se caracteriza por tensiones que tienen su origen en la reciente historia política del país. Las manifestaciones que provocaron la caída del régimen y la reconfiguración de las relaciones de poder han puesto las bases de un nuevo proceso histórico que despierta en el país grandes esperanzas de cambio y de renovación. Gracias a la fuerza de estos cambios, es ahora legítimo darle voz a una serie de aspiraciones sociales y económicas, antes reducidas casi al silencio.

La reivindicación de la ciudadanía pone en evidencia la condición de los grupos desfavorecidos de la sociedad, ocupados por problemas urgentes de supervivencia cotidiana. Se trata de dificultades concretas que encuentran sus motivaciones en las importantes tasas de desempleo y empleo precario y también en la falta de servicios accesibles. La dificultad de la transición hizo que los esfuerzos se centrasen en el ámbito institucional para establecer las bases de la democracia y es tiempo de dar respuestas contundentes a las demandas socio-económicas de la población y a sus reivindicaciones por la revolución (Hibou, Meddeb y Hamdi, 2011). La cuestión social en el Túnez de los próximos años vendrá seguramente marcada por la fractura social con relación a las posibilidades de reproducción económica, a la accesibilidad a los servicios, a la formación y al desarrollo comunitario. Los problemas de cohesión social pueden constituir un obstáculo importante para la nueva marcha democrática tunecina.

Por lo tanto, es en este marco de prioridades en el que el trabajador social puede ser un actor imprescindible de la nueva dinámica social, jugando un papel activo en la promoción de la participación y del desarrollo humano. Su for- mación y capacitación ha de orientarse a restaurar los futuros equilibrios sociales. Veremos a continuación cómo evolucionó dicha formación y los retos que tiene en la actualidad para contribuir a la consolidación de una sociedad solidaria que disfruta de sus derechos e intenta alcanzar una vida normalizada.

La formación en materia de Trabajo Social en Túnez se inicia en 1964, año de creación de la Escuela Nacional de Servicios Sociales, con una orientación clara hacia las metodologías del desarrollo y un perfil de profesionales llamado «Agentes de intervención». El cambio se produce en 1968, cuando un grupo de trabajadores sociales quebequenses inició las enseñanzas según el modelo quebequense de Trabajo Social, planteándolo como disciplina genérica de intervención basada en el método de caso, en el trabajo de grupos y en la organización comunitaria, partiendo de que las problemáticas que legitiman la acción se fundamentan en situaciones de desequilibrio, desorganización social, marginalidad y dependencia. En este marco, los entonces llamados agentes de intervención debían tener un perfil «polivalente» para resolver cualquier problema social específico. La principal crítica dirigida a este modelo de formación es la omisión de aquellos componentes estructurales causales de las situaciones problemáticas. Es por ello que en los años setenta surgió el debate sobre la necesaria concepción de un modelo autóctono de intervención social, adaptado a las problemáticas sociales de Túnez y a sus prioridades de acción. En 1975, la escuela pasa a denominarse Instituto Nacional de Trabajo y Servicio Social y crea nuevos itinerarios para formar a nuevos profesionales en materia de inspección del trabajo y administración de asuntos sociales. Hasta 1981, el instituto dejó de formar a los llamados «asistentes sociales» antes de retomar dicho itinerario y consolidar el debate sobre su importancia mediante el reconocimiento de tres niveles de legitimidad: la institucional (profesionales contratados para cumplir una tarea); democrática (cubrir las necesidades sociales de los usuarios) y legitimidad por competencias (capacidad de resolver problemas complejos). En 1986, la entidad cierra sus puertas y deja la plena competencia de formación al Instituto Nacional de Trabajo que se convierte más adelante en el Instituto Nacional de Trabajo y Estudios Sociales (Selmi, 2009). 
Es de resaltar el posicionamiento del Instituto, dentro de la estructura institucional tunecina. En efecto, el centro tiene una doble tutela: institucional, por el Ministerio de Asuntos Sociales; y académica, por el Ministerio de Enseñanza Superior e Investigación Científica, asumida directamente por la Université de Carthage. Aun así, el Instituto tiene personalidad civil y mantiene su independencia financiera y administrativa. Es reseñable esta peculiaridad del organismo en comparación con las Facultades y los Departamentos de Trabajo Social en España, cuya gestión es competencia de las universidades: esto permite a la formación en Trabajo Social disponer de las mismas oportunidades y recursos ofrecidos a las demás disciplinas. En el caso del Instituto, al encontrarse fuera de las instalaciones y las estructuras universitarias, los recursos son más limitados tanto a nivel administrativo como de formación e investigación.

La evolución de las enseñanzas, descrita más arriba, se refleja en los itinerarios ofrecidos por el Instituto Nacional de Trabajo y Estudios Sociales, pues integra estudios de Trabajo Social y otros estudios sociales y socio-laborales (ciencias del trabajo), que permiten la mutua transferencia de «buenas prácticas». Cuenta con un Departamento de Trabajo Social (denominado Service social) y oferta programas formativos siguiendo un esquema de titulaciones homologables a nivel internacional, y en concreto, en el Espacio Europeo de Educación Superior o «Acuerdos de Bolonia»: el esquema tunecino de diplomas de Licenciatura (3 años), Máster (2 años) y Doctorado (LMD). El instituto oferta programas formativos específicos de Grado en Trabajo Social (llamado Licence en Service Social), que cumplen los estándares internacionales, aunque con una división entre Grado «fundamental» y Grado «aplicado», uno con carácter teórico y otro enfocado al ámbito profesional: una doble oferta generalizada en todo el sistema de LMD tunecino. El Instituto Nacional de Trabajo y Estudios Sociales asume también enseñanzas de otros programas de grado, cercanos al Trabajo Social, denominados «Administración Social». Desde el curso 2012-2013, oferta programas de posgrado: Máster de investigación y Máster profesional en intervención social.

En función de esta oferta formativa, y más allá de los conocimientos adquiridos por los/as estudiantes, hemos de avanzar en la reflexión sobre sus competencias y actitudes como elementos esenciales en el perfil profesional del trabajador social. De forma general, se cuestiona la capacidad del sistema educativo tunecino a asegurar la integración social de los estudiantes ante los desafíos de sus contextos vitales (Hamami, 2012). Esta premisa se aplica al alumnado de Trabajo Social que no alcanza el desarrollo óptimo de sus valores de ciudadanía, del compromiso con la solidaridad y de sus habilidades pragmáticas, cuestión indisociable de la difícil evolución de las libertades en Túnez.

A nivel de las prácticas de grado, el instituto tiene a su disposición una red de organismos sociales, públicos principalmente, con capacidad para que los estudiantes realicen estancias de Practicum bajo la supervisión formativa de un tutor pedagógico y un tutor profesional. La conexión del Instituto Nacional de Trabajo y Estudios Sociales con su entorno institucional y social se apoya en las relaciones del profesorado con los trabajadores sociales y responsables de las diferentes entidades ligadas a la acción social; es lo que posibilita y favorece el programa de prácticas profesionales del alumnado.

A nivel internacional, el Instituto mantiene relaciones a través de convenios de colaboración con organismos como UNICEF o la Asociación Tunecina de Desarrollo Sostenible. No menos importantes son las que mantiene, dentro del ámbito de la cooperación internacional y a través de varios convenios, con el Institut Régional Européen des Métiers de l'Intervention sociale (ERIS-Francia), la École de Travail Social de Moncton-Canadá o el Institut des Sciences du Travail-Université Libre de Bruxelles. De forma generalizada al ámbito académico tunecino, el recurso a la cooperación fue y sigue siendo inevitable para los investigadores. Por factores de idioma y afinidad, la aportación francesa y quebequense es la más destacable y sirve tanto como puerta de acceso a una red académica más amplia como de reconocimiento para los universitarios tunecinos. A pesar de ello, la investigación científica sigue siendo marginada en el campo universitario tunecino y le cuesta desprenderse del peso de varias décadas durante las cuales la ciencia formó parte considerable de la ecuación del poder (Siino, 2004).

La dimensión de cooperación científica es de especial relevancia en el contexto tunecino actual. Como dice Schaefer (2009), con el obje- 
tivo de mejorar el alcance de su formación, el trabajador social debe ir al encuentro de sus colegas profesionales para favorecer la construcción de saberes basados en la experiencia y el intercambio de ideas y vivencias. La movilidad de los profesionales del Trabajo Social constituye un paso crucial para descubrir nuevos enfoques y realidades. Es por ello por lo que resulta importante, hoy más que nunca, avanzar en la confección de una «pedagogía del encuentro», dirigida a acercar las perspectivas de acción mediante el compromiso social y solidario de las universidades, en pleno diálogo con las contrapartes locales y favoreciendo el trabajo dentro del eje público-privado-civil (Aboussi, 2010). En la cooperación Norte-Sur, es importante darle máxima prioridad al principio de la reciprocidad (Favreau, Fréchette y Lachapelle, 2009): dentro de un paradigma horizontal de transferencia de conocimiento, las dos orillas del mediterráneo pueden retro-alimentarse. Es en este marco donde se inserta el proyecto SEPISE-INTES cuyas fases y resultados se describen a continuación.

\section{Descubriendo de cerca la realidad del Trabajo Social en Túnez}

Tras una fase preparatoria en la que el equipo del proyecto recopiló varios datos sobre el contexto sociopolítico y educativo en Túnez, la visita a la capital se planteó con la doble finalidad de realizar un diagnóstico del funcionamiento de la titulación en Trabajo Social en Túnez y descubrir la realidad de la profesión con la compañía del profesorado del Instituto Nacional de Trabajo y Estudios Sociales.

La distribución territorial de las instituciones de trabajo social está centralizada en varios ministerios y direcciones generales. En el ámbito regional y local, existen las unidades locales de promoción social y algunas intervenciones asociativas cuya mayor dificultad es la falta de financiación y/o de mecanismos de dirección estratégica. Los niveles prioritarios de actuación se centran en Infancia, Mujer y Familia, a través de varios programas dedicados principalmente a la integración social y la formación no reglada, ya que la tasa de absentismo escolar es bastante alta en algunos barrios, fuertemente caracteriza- dos por la pobreza y la vulnerabilidad de la población ${ }^{2}$. En este mismo contexto, nacen y se multiplican problemas como la violencia de género, la drogadicción o el alcoholismo que no suelen ser abordados con igual ímpetu.

La conexión del Instituto con esta estructura de servicios y su relación con los profesionales se refleja en el contenido y organización de las prácticas. La formación y aprendizaje del alumnado implica necesariamente un proceso en el que es muy relevante la participación de los tutores profesionales en el seguimiento de los estudiantes y la implicación del profesorado en la supervisión académica. El alcance de las prácticas y su incidencia en el desarrollo de destrezas del alumnado es motivo de preocupación de los diferentes actores implicados en este proceso.

En efecto, la coordinación y seguimiento durante el periodo de formación práctica, entre los agentes institucionales (tutores de prácticas) y el profesorado, parecen insuficientes para establecer los acuerdos necesarios en relación a los objetivos que el alumnado debe alcanzar y los contenidos que debe desarrollar en el período formativo de prácticas. Para ello, se manifiesta la necesidad de contar con un dispositivo formal mediante el cual se pueda desarrollar una relación pedagógica sólida entre los componentes del proceso. Se hace imprescindible formalizar convenios entre el Instituto Nacional de Trabajo y Estudios Sociales y las diferentes entidades públicas y privadas que acojan a los estudiantes en prácticas. Igualmente, se plantea el diseño de documentos e instrumentos de registro comunes y homogeneizados que permitan facilitar el seguimiento y evaluación del desarrollo de las prácticas y el logro de objetivos.

Estas dificultades ponen de manifiesto otra cuestión más compleja, compartida con el Trabajo Social en España, que abre un debate interesante sobre la relación entre teoría y práctica. Se cuestiona en específico el mecanismo de supervisión del aprendizaje teórico-práctico del alumnado. Sí es cierto que la mayoría de los tutores profesionales son egresados del Instituto y pueden transmitir su experiencia en la materia, pero quedan por definir aquellos instrumentos que facilitarían la inmersión de los estudiantes en la realidad de la profesión.

2 Túnez ocupa el puesto $90^{\circ}$ en el Índice de Desarrollo Humano de 2014. Véanse los detalles sectoriales en este enlace: http://hdr.undp.org/en/countries/profiles/TUN 
Como indicamos en la primera parte, la doble tutela del Instituto, y especialmente su relación con la Universidad de Cartago, no tiene un valor añadido, ya que no se aprovechan los recursos existentes para apoyar la formación en Trabajo Social. Esta premisa se plasma en la ausencia de mecanismos para orientar y asesorar en materia de inserción laboral. Aunque hemos de cuestionar también en España la eficacia de dichos dispositivos. Quizás sea una realidad compartida, esa falta de seguimiento de los titulados en cuanto a sus experiencias laborales, proyectos personales o estudios de Posgrado que emprenden. Siguen siendo escasas las iniciativas de orientación e información sobre los sectores con mayor número de contrataciones o yacimientos de empleo para el Trabajo Social. El Instituto Nacional de Trabajo y Estudios Sociales y el Ministerio de Asuntos Sociales, como principal empleador de trabajadores sociales en Túnez, podrían diseñar una estrategia armonizada para definir las necesidades en materia de recursos humanos y orientar la formación hacia ello, contemplando aquellos factores que limitan el acceso al empleo, como la centralización territorial o la marginalización del Trabajo Social. De igual modo, el papel del sector privado está siendo relegado a un segundo plano: es cierto que posee escasa incidencia sobre las contrataciones de los trabajadores sociales, pero ello es debido a la escasez de subvenciones y la falta de una concepción clara y estratégica de la gestión público-privada de los servicios sociales.

Estas observaciones se insertan en un marco de debate más amplio sobre la profesión de trabajador social en Túnez, cada vez más compleja ante la evolución del sistema de organización de servicios, la implicación de varias redes y la regulación cada vez mayor de la práctica. Por ello se intenta, desde 2008, elaborar un código deontológico por parte de la Asociación Tunecina de Servicios Sociales (ATSS), creada en el seno del Instituto Nacional de Trabajo y Estudios Sociales, aunque con ciertas dificultades para unificar los criterios y las referencias a tomar en cuenta respecto a la práctica (Selmi, 2009). Para ello, en la reflexión sobre la formación del trabajador social, los diferentes responsables a nivel nacional, regional y local, sugieren la participación de los estudiantes en reuniones para poner en marcha programas de promoción social, observación de fenómenos sociales y trabajo directo en «casos sociales», lo que les permitiría adquirir competencias para saber sistematizar todos los conocimientos y orientarlos a la práctica adecuada en una situación real (Jarray, 2009). Estando el país en una fase de reconstrucción, es un momento de diálogo y consenso en todos los ámbitos y quizás sea la ocasión de refundar una política social cuyos pilares dibujen claramente el modelo de Trabajo Social y el perfil de sus profesionales.

\section{Fase de intercambio sobre Trabajo Social en Granada}

La segunda parte del proyecto consistió en una visita de trabajo del profesorado del Instituto Nacional de Trabajo y Estudios Sociales a la ciudad de Granada. El objetivo fue ofrecer información en profundidad sobre la concepción española del trabajo social — como disciplina y como campo profesional- en el marco europeo e internacional, así como la organización universitaria de estos estudios, con atención específica a las prácticas formativas de trabajo social en instituciones de servicios sociales y otros servicios del bienestar. Se puso hincapié en la organización de las mismas, el marco institucional y los acuerdos que las hacen posibles, la estructura de servicios sociales básicos y específicos, las demás instituciones del bienestar que emplean a trabajadores/as sociales (sistema de salud, de justicia, de empleo, etc.), los roles de los actores participantes, así como otros aspectos sobre coordinación en diferentes niveles, materialización de los programas de prácticas, procesos y técnicas de tutoría, supervisión, coordinación y evaluación.

Para ello, se concretaron los ámbitos de trabajo abarcando en primer lugar un nivel administrativo, sobre la gestión universitaria de la titulación, del intercambio académico y de las prácticas profesionales; un nivel docente-investigador, sobre la enseñanza en el grado y el posgrado, su relación con la investigación y la presencia de los/as profesionales de los servicios sociales en las áreas académicas e investigadoras de la facultad; y finalmente, un nivel institucional de la intervención social, centrado en visitas a instituciones y espacios de reflexión y análisis.

Las Jornadas combinaron visitas a los establecimientos de la Universidad, en donde los responsables explicaron e intercambiaron información con la delegación del INTES. Desde la 
perspectiva de las necesidades detectadas junto a las propias preferencias de la visita tunecina, las sesiones de reflexión se organizaron para pausar los avances en la visita, permitiendo analizar comparativamente las organizaciones. Esta estrategia reveló que muchas de las dificultades encontradas en el Instituto Nacional de Trabajo y Estudios Sociales escapaban a las capacidades reales del grupo y su organización, y tenían que ver con la propia legislación o la configuración del sistema universitario en Túnez.

Por otra parte, se diseñaron visitas al terreno, a los servicios sociales pertenecientes a la Junta de Andalucía, a la Diputación de Granada, al Ayuntamiento de la ciudad y a organizaciones no gubernamentales de acción social. Se perseguía dar una visión completa, desde el punto de vista institucional, de la prestación de servicios en los distintos niveles territoriales que convergen en el municipio y, al mismo tiempo, de la participación del sector privado no lucrativo, colaborador del sistema público de servicios sociales. Aunque es cierto que existe, de manera creciente, una oferta privada mercantil en el sector socio-sanitario, la elección de centrarse sólo en organizaciones no gubernamentales perseguía acercar en lo posible ambas realidades, mostrando sectores de intervención posibles en Túnez, en el momento actual.

Con anterioridad, la delegación tunecina había expresado su interés por determinados colectivos y áreas de intervención, que fueron tenidos en cuenta en la programación. Se mostró un ejemplo de planificación y coordinación interadministrativa, del Área de Familia y Bienestar de la Diputación de Granada. Se eligieron dos recursos especializados en materia de drogodependencias y adicciones, de carácter ambulatorio e internamiento, ambos pertenecientes a la Junta de Andalucía: el Centro Provincial de Drogodependencias, que tiene desconcentrada su gestión en la Diputación de Granada y una comunidad terapéutica. Se mostró un centro municipal de servicios sociales y el entorno del barrio, en uno de los ocho distritos de la ciudad. Finalmente, Caritas Diocesanas representó al sector no lucrativo, y se visitaron algunas de las instalaciones para el desarrollo de programas de inclusión social.
De esta manera, se concretaron los objetivos que debían de priorizarse en el diseño de las actividades programadas. Por una parte, el conocimiento de la formación teórica y práctica en el ámbito del trabajo social. En segundo lugar, la investigación en trabajo social; tanto la forma de articular los grupos de investigación como la conexión nacional e internacional con otros colectivos universitarios o de instituciones superiores de enseñanza, dedicados al análisis de los problemas sociales. También se fijó como objetivo mostrar y analizar el modelo de prácticas de trabajo social y su articulación con la formación teórica, lo que abarcaba los dominios de intervención profesional y el sistema público y privado de atención, a disposición de la ciudadanía con necesidades específicas en España.

En todo caso, en la programación de la misión se habían fijado como principios rectores los fines y valores de la cooperación internacional: el inter-aprendizaje y reforzamiento mutuo, la educación-formación comparada, el aprendizaje colaborativo y la comunicación basada en el reconocimiento y respeto mutuo. La convivencia intensa a lo largo de los días de la misión ofreció múltiples oportunidades de poner en práctica tales principios, que contrastaban realidades religiosas, culturales, gastronómicas y sociales muy diversas. El mutuo conocimiento progresivo entre el profesorado de ambas instituciones se reforzó al compartir los objetivos comunes de mejorar el funcionamiento de las instituciones y de sus prácticas.

Puede considerarse como un ejemplo de aprendizaje mutuo el último taller de las jornadas que se centró en la innovación y metodología docente. La inquietud por la mejora de la enseñanza y la búsqueda de caminos para comprometer al alumnado en su propio aprendizaje y formación fueron preocupaciones compartidas desde ambas orillas, así como la reflexión y análisis sobre estas problemáticas.

Antes de concluir la fase de visita a Granada, se organizó una sesión de evaluación, precedida de un análisis común sobre el desarrollo del proyecto y de las perspectivas de futuro. Se combinaron varias técnicas y se realizó también una última valoración personal y anónima, para dar total libertad de expresión ${ }^{3}$. Esta reunión sirvió

3 Pasadas unas semanas y redactado el informe de recomendaciones y buenas prácticas de Trabajo Social, se hizo una devolución del proyecto, en este caso utilizando la multimedia para salvar la distancia geográfica entre los dos equipos de trabajo. 
igualmente para hacer una síntesis de los diferentes aportes del proyecto en materia de interaprendizaje y análisis comparado, llegando a afirmar que los contextos son diferentes pero las dificultades son iguales. Quizás la ventaja que tiene la formación en España es disponer de más recursos y herramientas de trabajo como la incorporación de las tecnologías de información y comunicación (TIC), el acceso abierto a la información, la movilidad de estudiantes y sobre todo mayores incentivos a la investigación en comparación con Túnez. Las futuras líneas de colaboración con el Instituto Nacional de Trabajo y Estudios Sociales se centrarán en dichos ámbitos.

\section{Conclusiones}

Una vez evaluado el proyecto, se puede afirmar la pertinencia de los objetivos que sirvieron de guía durante su ejecución, y que han sido: recabar información para poder realizar un diagnóstico veraz; ofrecer información en profundidad sobre el Trabajo Social en España, como disciplina y como profesión; el intercambio de información y de conocimientos; y la exploración de vías de cooperación permanente entre ambas instituciones que garanticen la permanencia y continuidad de las acciones emprendidas.

Al mismo tiempo, el desarrollo del proyecto ha sufrido un proceso de adaptación, durante su ejecución. Los aspectos de «consultoría» (en sus tareas de «asistir» y «aconsejar») han ido pasando a segundo plano, enfatizándose en cambio la dimensión del «inter-aprendizaje», entre la Universidad de Granada y el Instituto Nacional de Trabajo y Estudios Sociales, que contrasta dos situaciones institucionales y dos experiencias de organización, formación teórico-práctica e investigación en los dominios del Trabajo Social«Service Social».

La cooperación interuniversitaria se ha mostrado como una eficaz herramienta de colaboración horizontal entre instituciones de enseñanza superior. La trayectoria secular de la Universidad de Granada permite tomar decisiones en el ámbito educativo o en el de gestión universitaria, pero no son directamente aplicables como tales al ámbito tunecino del Instituto Nacional de Trabajo y Estudios Sociales. Las recomendaciones materializadas en una guía de buenas prácticas - entregada como parte de los productos acordados - reflejaron la trayectoria del trabajo social en España y fueron presentados como formas óptimas de ejecutar un proceso que podría servir de modelo para otras organizaciones y en un contexto determinado.

Sin embargo, dichas prácticas recomendadas deben ser adaptadas, pues no son estáticas, sólo deben promover nuevas ideas o sugerir adaptaciones y proporcionar una orientación sobre la manera más efectiva de visibilizar los diversos impactos en la Universidad. Cuando hablamos de buenas prácticas estamos haciendo referencia a la calidad y a la eficiencia dentro de las Universidades. Y esto tiene que ver con la gestión y los procedimientos, pero también con la satisfacción de las necesidades demandadas por la comunidad universitaria y la superación de sus limitaciones. Los contextos son determinantes para modular la eficacia de las acciones emprendidas; de ahí la necesidad de relativizar el impacto que la asistencia técnica podría tener.

Una ventaja del uso de metodologías participativas es el reconocimiento de la institución de referencia en las distintas fases del proyecto, lo que permitió hacer suyo desde el principio la valoración de las necesidades y carencias detectadas. El profesorado del Instituto Nacional de Trabajo y Estudios Sociales ha participado activamente en el mismo, en la detección y análisis de la situación, lo que permitió amortiguar las diferencias culturales en los diagnósticos del equipo sobre la institución local.

Para finalizar, siguiendo con la perspectiva del aprendizaje mutuo, estos proyectos de colaboración nos permiten tomar contacto con la lógica del trabajo social en culturas diferentes. Para trabajar en España con comunidades musulmanas por ejemplo, la cultura islámica debe reflejarse en la práctica del trabajo social, de tal manera que las políticas y los programas tengan en cuenta los aspectos culturales de las comunidades islámicas. Es necesario seguir explorando esta línea de trabajo, observación y colaboración, a través de éste $\mathrm{u}$ otro tipo de proyectos universitarios, que permitan colmar las muchas lagunas que existen en el área de Trabajo Social, tanto de conocimientos, como de habilidades y valores.

La continuidad y la apertura de nuevas vías de colaboración y entendimiento, junto al mantenimiento de las acciones emprendidas nos darán luz sobre las ventajas de la cooperación interuniversitaria en la que se reconoce el liderazgo y prestigio de los actores beneficiarios del proyecto; y esto sólo el tiempo podrá acreditarlo. 


\section{Referencias bibliográficas}

Aboussi, M. (2010). Le travail social international face aux défis de l'efficacité et du développment participatif. Travail-Emploi-Formation, 9 , 129-139.

Castro Martínez, E., Fernández de Lucio, I., Pérez Marín, M. y Criado Boado, F. (2008). La transferencia de conocimientos desde las humanidades: posibilidades y características. Arbor Issue, 732, 619-636.

Selmi, C. (2009). Code de déontologie des travailleurs sociaux en Tunisie, quels effets sur la profession. Actes du III ème congrés international intervention sociale et développment, quelles réferences pour les pratiques. Hammamet-Túnez.

Favreau, L., Fréchette, L. y Lachapelle, R. (2009). Cooperation Nord-Sud et développment, le défi de la réciprocité. Montréal: Presses de 1'Université du Québec.

Hamami, A. (2012). Éducation et Cohésion Sociale en Tunisie. African Sociological Review, 16 (2), 56-80.

Hibou, B., Meddeb, H. y Hamdi, M. (2011). La Tunisie d'après le 14 janvier et son économie politique et sociale. Copenhague: Réseau Méditerranéen des Droits de 1'Homme.

Jarray, F. (2009). Pour un référentiel professionnel de l'intervenant social de première ligne en Tunisie. Actes du III Congrès International de l'AIFRIS, 21-24 de abril. Hammamet-Túnez.

Lorente, B. y Zambrano, C. (2010). Reflexividad, Trabajo Comunitario y sensibilización de derechos. Cuadernos de Trabajo Social, 23, 85-102.

PNUD. (2014). Human Development Report-Tunisia. Recuperado de: http://hdr.undp.org/en/countries/profiles/TUN (Consultado el 29/10/2014).

Schaefer, G. (2009). Perspectives Internationales en Travail Social. En B. Destremau, E. Parpaillon, C. Rollet y G. Schaefer, Le social dans la mondialisation (pp. 59-81). Rennes: Presses de 1'EHESP.

Siino, F. (2004). Science et pouvoir dans la Tunisie Contemporaine. Paris: Khartala-IREMAM. 